Ann. Génét. Sél. anim., 1976, 8 (2), 273-283.

\title{
VARIABILITÉ ET HÉRITABILITÉ DES PERFORMANCES DE CROISSANCE CHEZ LA TRUITE ARC-EN-CIEL (SALMO GAIRDNERII RICHARDSON).
}

\author{
B. CHEVASSUS
}

avec la collaboration technique de A. M. Escaffre et F. Herioux

Laboratoire de Physiologie des Poissons,

Centre national de Recherches zootechniques, I. N.R. A.,

78350 Jouy en Josas

\section{RÉSUMÉ}

Trois composantes de la variabilité des performances de croissance (intrafamille de pleinfrères, interfamilles intrasouche, intersouches) ont été étudiées chez la Truite arc-en-ciel de o à 5 oo jours.

Les résultats obtenus pour la variabilité relative (coefficient de variation) sont les suivants :

- La composante intrafamille, faible à l'éclosion, augmente rapidement au début de l'alimentation. Les valeurs maximales sont atteintes vers le $3^{\mathbf{e}}$ mois (coefficient de variation de l'ordre de $40 \mathrm{p}$. I 00 pour le poids et $14 \mathrm{p}$. Ioo pour la longueur). On observe ensuite une lente diminution de cette composante.

- La composante interfamilles est influencée initialement par des effets maternels (taille des œufs) qui disparaissent après le $2^{\mathrm{e}}$ mois. Elle se stabilise ensuite à environ ro p. roo pour le poids et $3 \mathrm{p}$. roo pour la longueur.

-- La composante intersouches est assez importante (de l'ordre de 20 p. roo).

L'héritabilité calculée à partir de la covariance entre plein-frères prend des valeurs assez faibles $(0,045$ à 0,375$)$.

Les distributions intrafamilles du poids sont sensiblement normales à l'éclosion puis acquièrent un caractère asymétrique (distribution de type Log-normal) qui disparaît graduellement à partir de 3 mois. La transformation logarithmique des données ne modifie pas sensiblement les estimations de l'héritabilité et du progrès génétique.

\section{I. - IN'TRODUC'TION}

Parmi les critères conditionnant la rentabilité de la salmoniculture, les performances de croissance jouent un rôle majeur. Au sein d'une espèce donnée, l'existence d'une importante variabilité de ces performances a incité certains auteurs à mettre 
en place une sélection visant à améliorer ce caractère (DONALDSON et al., I955). Les résultats obtenus, quoique positifs, sont cependant d'interprétation délicate, du fait de l'absence de population témoin et des variations des facteurs du milieu au cours de la sélection. Il est donc difficile d'évaluer, à partir de ces résultats, l'importance relative des facteurs génétiques et des facteurs du milieu dans le déterminisme de la variabilité observée. Pour répondre à cette question, Aulstad et al. (I972) ont réalisé une analyse du rôle de ces deux composantes de la croissance chez la Truite arc-en-ciel (Salmo gairdnerii RICHARDSON). Les valeurs obtenues pour l'héritabilité de la taille et du poids (rapport de la variance génétique additive à la variance phénotypique totale) sont de l'ordre de 0,20 et permettent d'envisager favorablement une amélioration génétique de la croissance.

Cependant, pour évaluer précisément l'espérance du progrès génétique, il est nécessaire :

- d'estimer la variabilité phénotypique totale existant dans l'espèce, en incluant les différences entre les nombreuses souches locales existant dans les piscicultures qui peuvent avoir acquis, du fait de leur isolement, des caractéristiques originales;

-- de préciser la structure des populations d'élevage et son évolution au cours du temps, afin de vérifier notamment la normalité de la distribution de la taille et $\mathrm{du}$ poids dans ces populations. Si cette hypothèse n'est pas satisfaite, il convient en effet de rechercher une transformation normalisante et d'estimer le progrès génétique sur la variable transformée.

Cette étude, portant sur la croissance de la Truite arc-en-ciel de o à 500 jours, résume les résultats relatifs à ces deux problèmes.

\section{II. - MATÉRIEL ET MÉTHODES}

La population étudiée est constituée de 8 souches issues de piscicultures françaises et danoises possédant un cheptel de géniteurs de l'ordre d'une centaine d'animaux entretenu sans apport extérieur depuis plusieurs générations. L'étude comparative de ces souches a été réalisée à la pisciculture de Montbard (Cote-d'Or) pour trois densités d'élevage différentes. (30, 60 et roo individus par bac). Les souches sont introduites au stade d'œufs œillés et sont élevées séparément dans des bassins de taille croissante $(201$, I6o 1, I ooo 1), et nourries ad libitum. I.es paramètres du milieu (débit, température de l'eau) sont homogènes pour l'ensemble des lots expérimentaux. En outre, on a pu disposer pour deux de ces souches d'un échantillon de reproducteurs qui ont permis de constituer I9 familles de plein-frères (I I d'une souches et 8 de l'autre), permettant ainsi de décomposer la variabilité en 3 composantes (intrafamille, interfamilles, intersouches). Cette deuxième étude a été réalisée au laboratoire de Physiologie des Poissons de l'I.N.R.A. (Jouy-en-Josas, Yvelines). Les paramètres mesurés sont le poids et la longueur standard. L'étude de la structure des distributions a été réalisée à I mois, 3 mois et Io mois sur l'ensemble des 19 familles, les données de chaque famille étant centrées et réduites en utilisant les paramètres de cette famille, Les tests de normalité utilisés sont le test de CHI 2 et les tests portant sur les coefficients d'asymétrie et d'aplatissement de Pearson (SNedecor, I956).

Pour estimer les composantes de la variance, on utilise un modèle aléatoire hiérarchique :

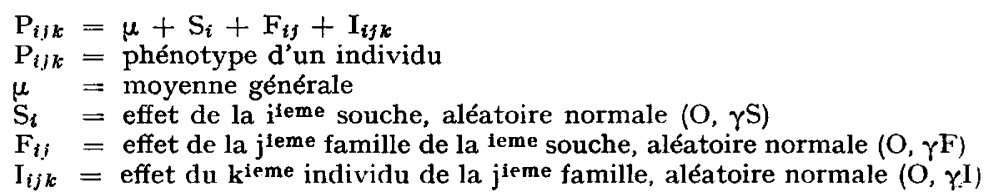


$\gamma_{\mathrm{S}}, \gamma_{\mathrm{F}}, \gamma_{\mathrm{I}}$ représentent respectivement les composantes intersouches, interfamilles intrasouche et intrafamille.

L'homogénéité des variances intrafamilles a été testée par la méthode de Bartlett.

La variabilité relative est mesurée par le coefficient de variation $\mathrm{C}=100 \times \frac{\sqrt{\gamma}}{\mu}$. Le calcul des paramètres génétiques utilise le modèle de Falconer (1960). Dans ce modèle, le double de la corrélation intraclasse $\left(\frac{\gamma_{F}}{\gamma_{F}+\gamma_{I}}\right)$ fournit une borne supérieure de l'héritabilité.

On a en effet, en négligeant les effets d'épistasie :

$$
\frac{2 \gamma_{F}}{\gamma_{F}+\gamma_{I}}=\frac{V_{A}+1 / 2 V_{D}+V_{M}}{V_{P}} \geqslant h^{2}=\frac{V_{A}}{V_{P}}
$$

avec $V_{\mathrm{A}}$ : variance des effets génétiques additifs;

$V_{D}$ : variance des effets génétiques de dominance ;

$V_{M}$ : variance des effets maternels;

$\mathrm{V}_{\mathrm{P}}$ : variance phénotypique totale.

L'crreur standard de l'héritabilité est tirée de Swiger et al. (I964).

\section{III. - RÉSULTATS}

\section{I. - Variabilité phénotypique}

L'évolution des composantes de la variabilité phénotypique du poids et de la longueur à différents âges est indiquée dans les figures I et 2.

\section{a) Composante intrafamille.}

Au stade œillé, la variabilité de la taille des œufs d'une même ponte est faible (4 à $6 \mathrm{p}$. IOO). Cette homogénéité intrafamille se maintient à 1'éclosion et pendant toute la résorption de la vésicule vitelline.

Au début de la nutrition, on assiste à une augmentation rapide de la variabilité, le poids moyen du lot restant sensiblement constant pendant la phase d'acquisition du comportement alimentaire. La variabilité relative atteint des valeurs maximales de l'ordre de $40 \mathrm{p}$. Ioo pour le poids et $\mathrm{I} 5 \mathrm{p}$. Ioo pour la longueur à partir de $\mathrm{I}, 5$ à $2 \mathrm{~g}(50 \mathrm{~mm})$.

Ces valeurs correspondent à des différences de taille considérables entre individus, l'étendue maximale observée étant $(0,99-5,26 \mathrm{~g})$ pour une famille de poids moyen 2,06 g.

On observe ensuite une lente décroissance de la variabilité relative jusqu'à des valeurs de l'ordre de 25 à $30 \mathrm{p}$. Ioo pour le poids et Io $\mathrm{p}$. I00 pour la longueur à I8 mois.

\section{b) Composante interfamilles intrasouche.}

La variabilité interfamilles reflète initialement la variabilité interfemelles de la taille des œufs. Cependant, ces effets maternels disparaissent graduellement, la corrélation taille des œufs-poids moyen du lot s'annulant assez rapidement (tab1. I). Néanmoins la variabilité interfamilles se maintient jusqu'à $\mathrm{r} 8$ mois à des valeurs de l'ordre de ro $\mathrm{p}$. Ioo pour le poids et 3 à $4 \mathrm{p}$. Ioo pour la longueur. 


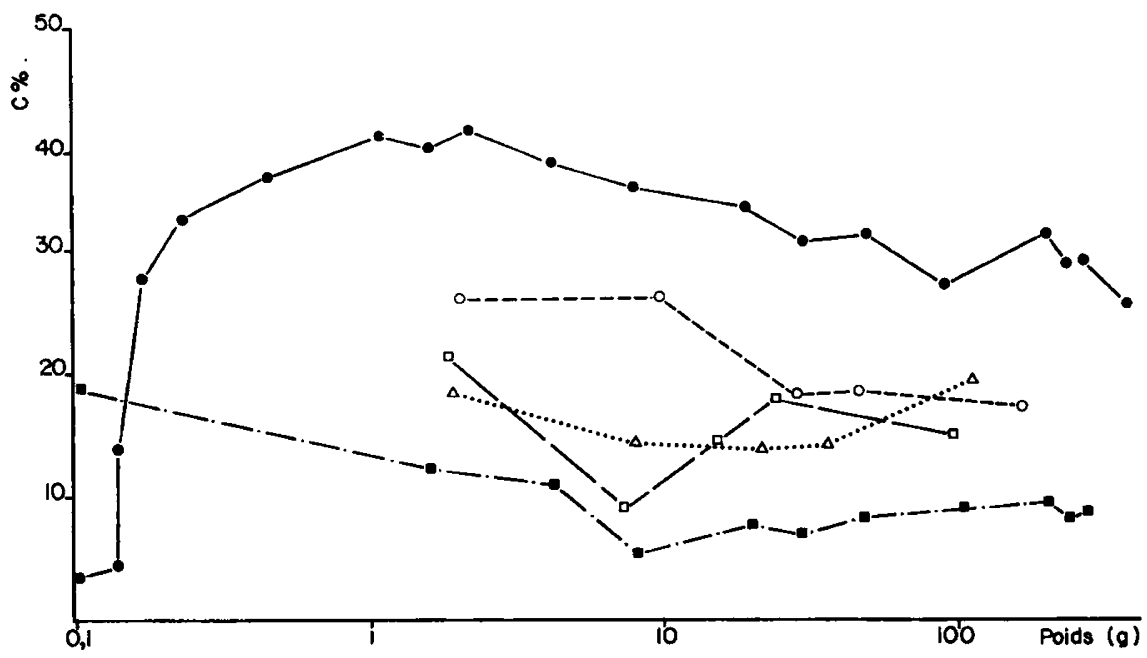

FIG. I. - Variabilité de la croissance pondérale.

I. volution du coefficient de variation du poids $\left(100 \times \frac{\text { écart-type }}{\text { moyenne }}\right)$ au cours de la croissance

Variability of growth in weight. Evolution of the coefficient of variation of weigth during growth

- Composante intra-famille

- Composante inter-familles intra-souche Between families within strains component

o Composante inter-souches (densité A) Between strains component (densité faible) (low density)

$\Delta$ Composante inter-souches (densité B) Between strains component (densité moyenne) (intermediate density)

$\square$ Composante inter-souches (densité C) Between strains component (densité forte)

(high density)
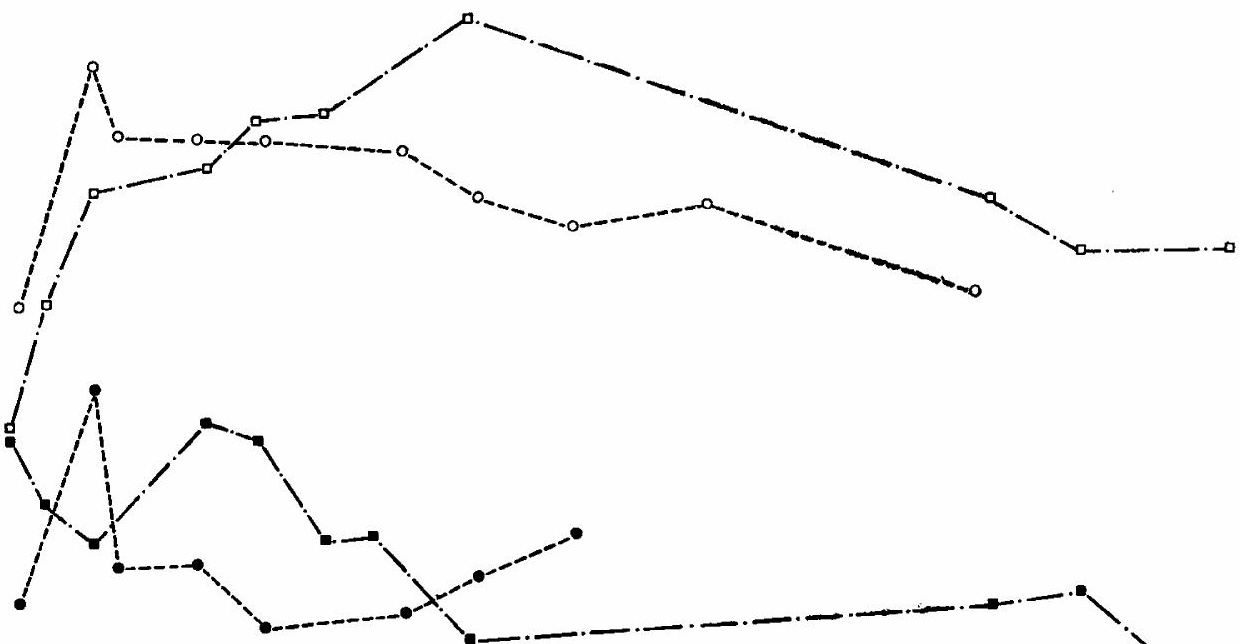

FIG. 2. - Variabilitê de la croissance en longueur pour deux souches $S 1$ et $S 2$ :

Evolution du coefficient de variation de la longueur au cours de la croissance

Variability of growth in length for strains $S 1$ and $S 2$ :

Evolution of the coefficient of variation of length during growth for two strains $S 1$ and $S 2$

$\mathrm{S} I \mathrm{O}$

$\left.\begin{array}{ll}S_{2} & 0\end{array}\right\}$

$\mathrm{S} I \bullet$

S 2 .
Within families component composante inter-familles intra-souche Between families within strains component 


\section{TABLEAU I}

Effet de la taille des aufs (corrélation volume moyen des œufs - longueur moyenne de la famille établie sur i9 familles)

Effect of egg size

(correlation average egg volume

- average lenght of the family)

\begin{tabular}{c|c|c|c|c}
\hline \hline Age (jours) & 30 & 70 & 95 & 135 \\
\hline \begin{tabular}{c} 
Longueur moyenne $(\mathrm{mm})$ \\
\hline $\begin{array}{c}\text { Corrélation } \\
\text { (longueur-volume } \\
\text { des } 0 \text { ufs) }\end{array}$
\end{tabular} & $+0,647 \mathrm{~S} 1 \%$ & $+0,50 \times \mathrm{S} 5 \%$ & $+0,089 \mathrm{NS}$ & $+11,050 \mathrm{NS}$ \\
\hline
\end{tabular}

c) Composantes intersouches.

L'étude des différences de croissance entre les différentes souches conduit à des estimations de la variabilité relative de l'ordre de $I_{5}$ à $20 \mathrm{p}$. Ioo pour le poids. Une analyse de variance à effets fixes réalisée sur la variable " poids à 450 jours " (tabl. 2) permet de mettre en évidence :

- des différences significatives entre souches ;

- une influence de la densité d'élevage, les densités élevées conduisant à des croissances plus lentes. Cependant, la variabilité relative des performances de croissance reste du même ordre aux trois densités ;

- l'absence d'interaction souche $x$ densité.

TABLEAU 2

Analyse de variance.

Effet souche et effet densité sur le poids à 450 jours

Analysis of variance

effect of the density and effect of the strain on body weight at 450 days

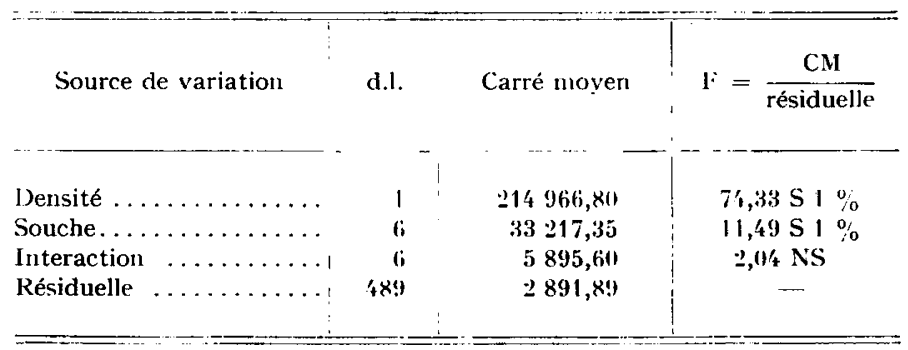

\section{2. - Structure des distributions}

L'étude de la structure des distributions du poids, réalisée à différents stades de la croissance est résumée dans le tableau 3. 
TABLEAU 3

Structure des distributions du poids à différents stades de développement Structure of weight distributions at various stages of development

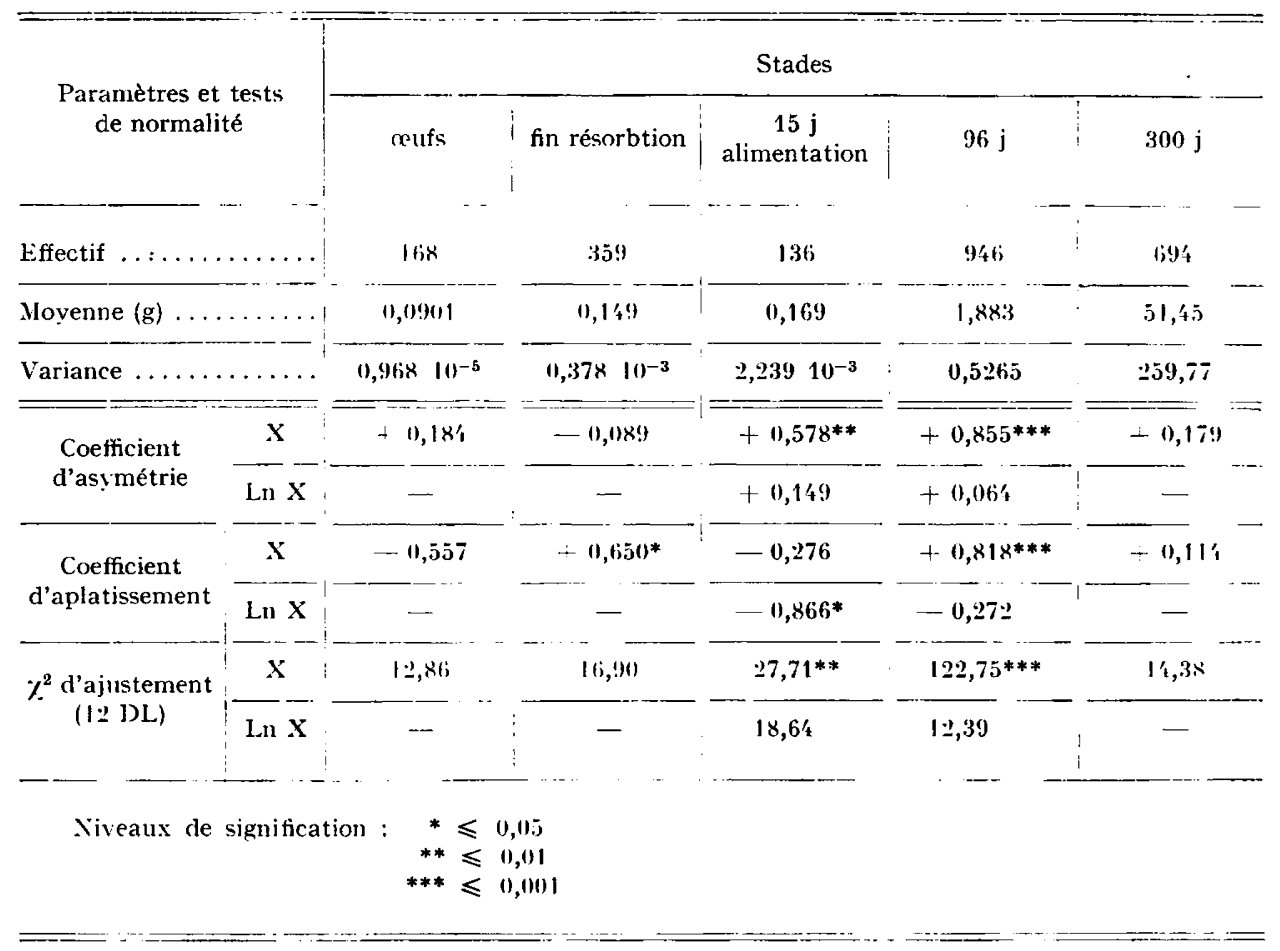

- Pour les œufs fécondés, les distributions sont de type unimodal symétrique et peuvent être considérées comme normales (fig. $3 \mathrm{~A}$ et tabl. 3 ).

- La résorption de la vésicule vitelline, qui se termine une trentaine de jours après l'éclosion, conduit à une légère augmentation de la variabilité. Les distributions restent cependant sensiblement normales pendant cette période (fig. $3 \mathrm{~B}$ et tabl. 3).

- La phase d'acquisition du comportement alimentaire se traduit, parallèlement à l'augmentation rapide de la variabilité, par l'apparition d'une asymétrie marquée dans la distribution (fig. $3 \mathrm{C}$ et tabl. 3). Une analyse plus fine du phénomène (fig. 4) montre que pendant cette période, il y a croissance rapide d'une fraction de la population, avec stagnation, voire amaigrissement des individus les plus petits, d'où création d'une dissymétrie. L'utilisation de la transformation $\mathrm{Y}=\operatorname{Ln} \mathrm{X}$ permet de normaliser la distribution de manière satisfaisante, les courbes devenant cependant légèrement platykurtiques.

- Ce caractère asymétrique des distributions s'accentue par la suite, son maximum coïncidant sensiblement avec le maximum de variabilité relative $(\mathrm{I}, 5$ à $2 \mathrm{~g}$ ). Les distributions sont alors franchement de type Log-normale (fig. 3 D), la transformation logarithmique permettant un excellent ajustement (tabl. 3). 


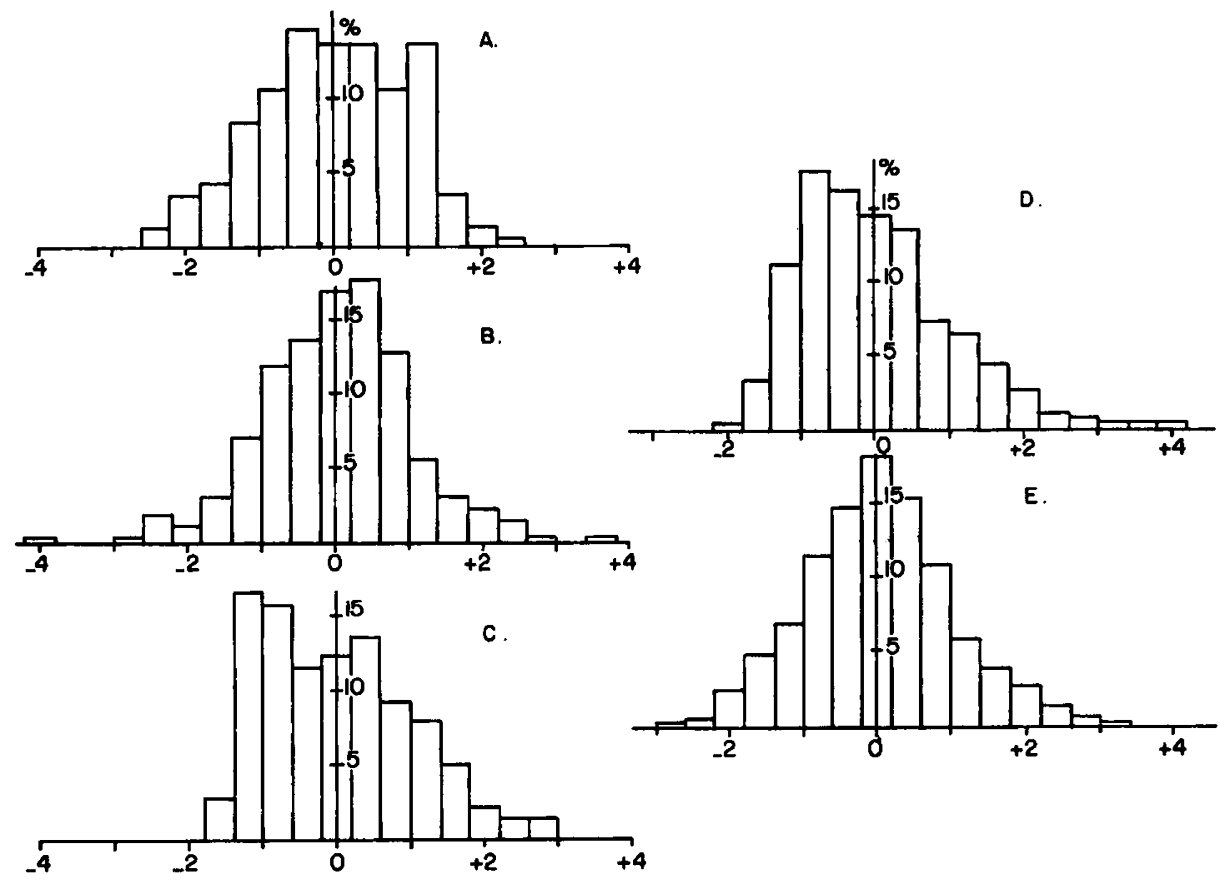

FIG. 3. - Structure des distributions du poids (variable centrée réduite)

Structure of weight distributions (standard measure)
A Stade œillé
Eyed stage
$B$ Fin de la résorption vésiculaire
C Début de l'alimentation
D 3 mois
E ro mois
End of resorption
Start of feeding
3 months
Io months

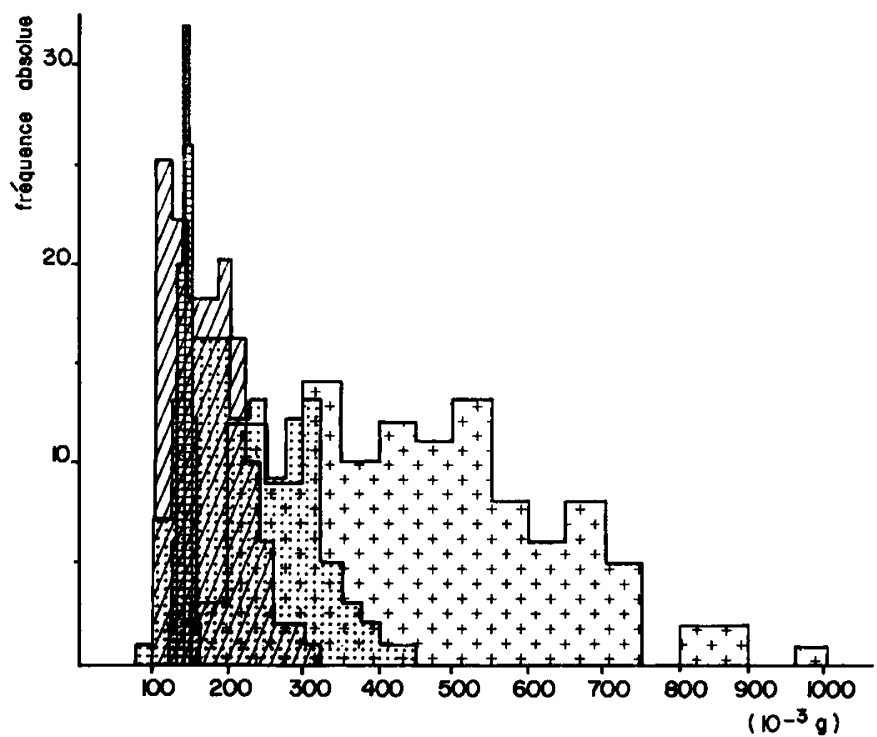

FIG. 4. - Évolution de la distribution des poids au cours des deux premiers mois Changes of weight distributions ditring the two first months

15 j après éclosion 15 days after hatching 42 $\mathrm{j}$ après éclosion 45 days after hatching : $+++63 j$ après éclosion 63 days after hatching 
- Au cours de la croissance ultérieure, on assiste à une diminution de l'asymétrie. A $50 \mathrm{~g}$, les différents tests ne permettent plus de déceler d'écarts à la normalité (fig. $3 \mathrm{E}$ et tabl. 3).

\section{3. - Héritabilité de la croissance}

Les données recueillies sur les I9 familles de plein-frères (effectif de l'ordre de $50 \pm$ Io) permettent de calculer l'héritabilité de la longueur et du poids à différents âges. Les estimations obtenues (fig. 5) sont du même ordre de grandeur pour ces deux paramètres et sont comprises entre 0,045 et 0,375 , l'erreur standard sur ces mesures étant de l'ordre de o, Ioo.

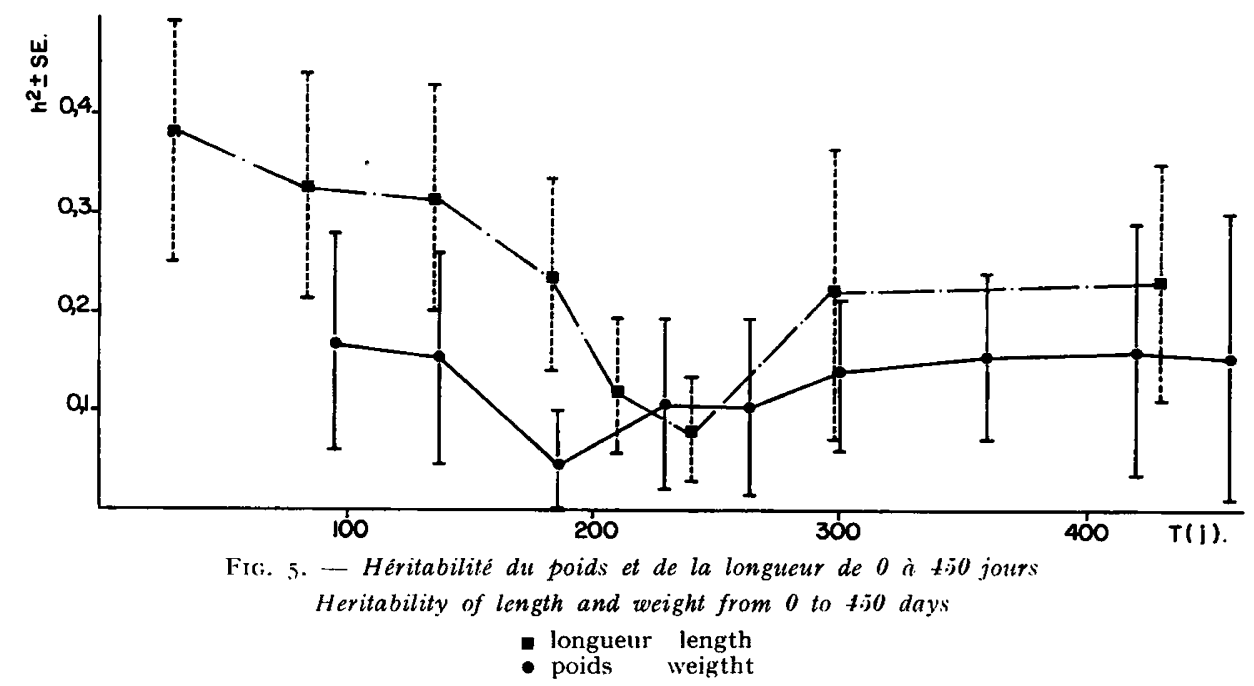

Pour tenir compte de l'asymétrie de certaines distributions, nous avons également effectué les calculs sur les données logarithmiques. Les corrections à apporter aux estimations de l'héritabilité se révèlent faibles (de l'ordre de $\pm 0,02$ ). I) même, l'estimation du progrès génétique sur la variable transformée n'apporte pas de modifications notables (corrections de l'ordre de \pm 5 p. I00).

\section{IV. - DISCUSSION}

\section{A. - Héritabilité de la croissance}

Less estimations obtenues pour l'héritabilité de la croissance sont voisines de celles obtenues par d'autres auteurs chez les Salmonidés (Auls'sad et al., I972; Ryman, I972) et les Cyprinidés (KIRPITSCHNIKov, I966). Il conviendrait cependant de préciser l'importance des effets de dominance, qui rentrent pour moitié dans notre estimation. Les données de Aulstad et al. (I972) indiquent que ces effets semblent relativement faibles. Il semble donc possible de retenir pour l'héritabilité 
de la croissance des valeurs de 0 , Io à 0,25 pour les espèces étudiées. Cependant plusieurs critiques sont à adresser au dispositif expérimental classique d'élevage comparatif en lots séparés.

- On ne peut affirmer que les différents lots soient strictement dans les mêmes conditions de milieu. Cette hétérogénéité du milieu s'ajoute aux différences d'origine génétique et conduit donc à surestimer l'héritabilité. Cependant les données de AUlstAd et al. (1972) indiquent que, dans un élevage soigneusement contrôlé, cette variabilité interbassins est faible.

- A l'intérieur d'un lot, les interactions sociales tendent à amplifier les différences existant entre les individus. Les données de Browy (1946) sur Salmo trutta indiquent clairement que la position hiérarchique d'un individu dans une population conditionne étroitement la croissance de cet individu, même dans un régime d'alimentation ad libitum. La suppression de ce facteur social, obtenue par des élevages individuels (NAKAmura et KASAHARA, I957 chez Cyprinus carpio ; PURdom, I972 chez les Pleuronectidés, Chevassus, $1976 \mathrm{chez}$ Salmo gairdnerii) conduit à une réduction considérable de la variabilité. Inversement, la mise en compétition de plusieurs groupes (MOAV, I974 sur Cyprinus carpio) conduit à augmenter considérablement les différences mesurées entre les mêmes groupes témoins conservés isolément. L'élevage en lots séparés, rendu nécessaire par l'impossibilité de marquer précocement les lots, conduit donc éventuellement à une sous-estimation de l'héritabilité du caractère.

- Enfin, l'existence d'interactions génotype-facteur social n'est pas à exclure a priori. Les facteurs génétiques impliqués dans la variabilité intralot, qui s'expriment à travers le facteur social, seraient alors relativement différents de ceux responsables de la variabilité interlots. La comparaison de ces deux composantes et l'estimation de l'héritabilité qui en découle seraient dans ce cas sans signification biologique. L'étude réalisée par MOAv (I974) conclut à l'absence de telles interactions chez la Carpe (Cyprinus carpio L.). Cependant 1'extrapolation de ce résultat aux Salmonidés semble difficile, leur régime et leur comportement alimentaire étant notablement différents.

Il convient donc d'être prudent en ce qui concerne la valeur des estimations obtenues pour l'héritabilité dans un tel schéma expérimental.

\section{B. - Structure des distributions}

L'apparition d'une asymétrie marquée des distributions dès le début de la nutrition semble résulter essentiellement des phénomènes de compétition entre les individus. En effet, les expériences d'élevage individuel conduisent généralement à des distributions symétriques (NAKAMURA et KASAHARA, I955, r957). La transformation logarithmique des données permet de normaliser ces distributions de manière satisfaisante pendant les premiers mois de croissance, mais ne modifie que très peu les estimations de l'héritabilité et de l'espérance du progrès génétique.

\section{C. - Variabilité phénotypique'}

Les estimations de la variabilité phénotypique du caractère rejoignent celles de GJEDREM (I975). L'existence d'une variabilité intersouches relativement importante pose le problème du schéma d'amélioration génétique à envisager chez les 
Salmonidés. La sélection massale fréquemment pratiquée et pour laquelle les estimations du progrès génétique sont encore imprécises pourrait, dans un premier temps du moins, être remplacée par une étude comparative des différentes souches disponibles et de leurs croisements, ce qui permettrait d'obtenir et de diffuser rapidement un progrès génétique notable.

\section{V. - CONCLUSIONS}

-- Dans un même milieu, la variabilité phénotypique des performances de croissance de la Truite arc-en-ciel est importante (coefficient de variation de l'ordre de 40 à $50 \mathrm{p}$. Ioo pour le poids à un âge donné).

- La variabilité à l'intérieur des familles de plein-frères représente la composante la plus importante de la variabilité totale.

- L'étude des composantes de cette variabilité phénotypique montre que la composante intrafamille de plein-frères est très supérieure à la composante interfamilles. Les valeurs de l'héritabilité des performances de croissance sont donc assez faibles $(0,045$ à 0,375$)$.

- Les différences de croissance entre souches sont également notables; une sélection basée sur le testage de souches est donc envisageable.

- Les distributions intralots sont de type Log-normale au début de la croissance mais peuvent être considérées comme normales à partir de $50 \mathrm{~g}$. La transformation logarithmique des données ne conduit cependant qu'à des corrections mineures en ce qui concerne les estimations de l'héritabilité et du progrès génétique.

Rę̧u pour publication en mai 1976.

\section{SUMMARY}

\section{VARIABILITY AND HERITABILITY OF GROWTH PERFORMANCES IN RAINBOW TROUT (SALMO GAIRDNERII RICHARDSON)}

Three components of variability of growth performances (Individual within full sib families ; between families within strains; between strains) was measured from o to 500 days in rainbow trout.

The results obtained for relative variability (coefficient of variation $\mathrm{C}$ ) are the following :

- The within families component, very small after hatching $(C=3$ to 4 p. Ioo) quickly increased at the start of feeding. Maximal values were obtained at 3 months $(C=40 \mathrm{p}$. Ioo for weight and $I_{4}$ p. Ioo for length) and decreased thereafter.

- The between families component was initially influenced by maternal effects (egg size) which disappeared after two months. After that, the mean values were ro p. Ioo for weight and 3 p. roo for length.

- The between strains component was rather large (about 20 p. 100).

The heritability, estimated on the basis of covariance between full sibs, was rather small $(0.045$ to 0.375$)$.

The within families distributions of weight were practically normal after hatching and then became asymmetrical (Log normal distribution). This asymmetry decreased slowly after 3 months. The logarithmic conversion of data did not substantially change the estimates of heritability and genetic progress. 


\section{RÉFÉRENCES BIBLIOGRAPHIQUES}

Aulstad D., Gjedrem T., Skervold H., 1972. Genetic and environmental sources of variation in lenght and weight of rainbow trout (Salmo gairdnerii). J. Fish. Res. Bd. Can., 29, 237-24I.

Brown M. E., I946. The growth of brown trout (Salmo trutta Linné). I. Factors influencing the growth of trout fry. J. Exp. Zool., 22, I18-r30.

Chevassus B., I976. Génétique et Aquaculture : Esquisse d'une méthodologie. Oceanis, 2, $187-218$.

Donaldson L. R., Olson P. R., 1955. Development of rainbow trout brood stock by selective breeding. Trans. Amer. Fish. Soc., 85, 93-IoI.

Falconer D.S., I960. Introduction to quantitative genetics. Oliver and boyd, Edinburgh.

GJedrem T., 1975. Possibilities for genetic gain in Salmonids. Aquaculture, 6, $23-29$.

Kirpitschnikov V.S., I966. Efficiency of mass selection and selection for relatives in fish culture. FAO Fish Reports, 44, 4.

Moav R., Wohlfarth G. W., 1974. Magnification through competition of genetic differences in yield capacity in carp. Heredity, 38, 181-202.

Nakamura N., Kasahara S., 1957. A study of the phenomenon of the tobi koi or shoot carp. III. On the results of culturing the modal group and the growth of carp fry reared individually. Bull. Jap. Soc. Sci. Fish., 22, 674-678 (in Japanese, with English summary).

Nakamura N., Kasahara S., I955. A study of the phenomenon of the tobi koi or shoot carp. I. On the earliest stage at which the shoot carp appears. Bull. Jap. Soc. Sci. Fish., 21, 73-76 (in Japanese with English summary).

Purdom C. E., I972. Genetics and fish farming. Laboratory Leaflet (New series), $\mathrm{n}^{\circ} 25$.

Ryman N., 1972. An analysis of growth capability in full sib families of salmon (Salmo salar L.) Hereditas, 70, $119-128$.

Snedecor G. W., 1956. Statistical methods, 5th ed. The Iowa state University Press, Ames, Iowa, U.S.A.

Swiger L. A., Harvey W. R., Everson D. O., Gregory K. E., Ig64. The variance of intraclass correlation involving groups with one observation. Biometrics, 20, 81 8-826. 